\title{
IncRNA H19 binds VGF and promotes pNEN progression via PI3K/AKT/CREB signaling
}

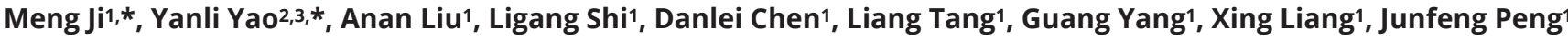 \\ and Chenghao Shao' ${ }^{1}$ \\ 'Department of General Surgery (Department of Pancreatic-Biliary Surgery), Shanghai Changzheng Hospital, The Second Military Medical University, \\ Shanghai, China \\ 2Glycochemistry \& Glycobiology Lab, Key Laboratory of Receptor Research, Shanghai Institute of Materia Medica, Shanghai, China \\ ${ }^{3}$ University of the Chinese Academy of Sciences, Beijing, China
}

Correspondence should be addressed to C Shao: shaochenghao_czyy@163.com

*(M ji and Y Yao contributed equally to this work)

\begin{abstract}
Pancreatic neuroendocrine neoplasms (pNENs) are endocrine tumors arising in pancreas and is the most common neuroendocrine tumors. Mounting evidence indicates IncRNA H19 could be a determinant of tumor progression. However, the expression and mechanism of $\mathrm{H} 19$ and the relevant genes mediated by $\mathrm{H} 19$ in pNENs remain undefined. Microarray analysis was conducted to identify the differentially expressed IncRNAs in pNENs. H19 expression was analyzed in 39 paired pNEN tissues by qPCR. The biological role of $\mathrm{H} 19$ was determined by functional experiments. RNA pulldown, mass spectroscopy and RNA immunoprecipitation were performed to confirm the interaction between H19 and VGF. RNA-seq assays were performed after knockdown H19 or VGF. $\mathrm{H} 19$ was significantly upregulated in pNEN tissues with malignant behaviors, and the upregulation predicted poor prognosis in pNENs. In vitro and in vivo data showed that $\mathrm{H} 19$ overexpression promoted tumor growth and metastasis, whereas H19 knockdown led to the opposite phenotypes. H19 interacted with VGF, which was significantly upregulated in pNENs, and higher VGF expression was markedly related to poor differentiation and advanced stage. Furthermore, VGF was downregulated when H19 was knocked down, and VGF promoted cell proliferation, migration and invasion. Mechanistic investigations revealed that $\mathrm{H} 19$ activated PI3K/AKT/CREB signaling and promoted pNEN progression by interacting with VGF. These findings indicate that $\mathrm{H} 19$ is a promising prognostic factor in pNENs with malignant behaviors and functions as an oncogene via the VGF-mediated PI3K/AKT/CREB pathway. In addition, our study implies that VGF may also serve as a candidate prognostic biomarker and therapeutic target in pNENs.
\end{abstract} Key Words

\section{Introduction}

Neuroendocrine neoplasms (NENs) arise from neuroendocrine systems and often occur in tissues, such as lung, pancreas and pituitary tissues (Ohki et al. 2014, Gao et al. 2018). However, due to the low incidence and the lack of suitable experimental model systems, the mechanisms underlying the development of NENs remain unclear (Ohki et al. 2014).
C) 2019 Society for Endocrinology Published by Bioscientifica Ltd. Printed in Great Britain 
Pancreatic neuroendocrine neoplasms (pNENs), occurring in the duodenal-pancreatic region and constituting $1-2 \%$ of pancreatic neoplasms, is the second most common pancreatic neoplasm (Yohanathan et al. 2019). pNENs include functional and nonfunctional tumors as well as tumors that may represent a manifestation of a hereditary neuroendocrine syndrome (Yohanathan et al. 2019).

pNENs may be benign or malignant. pNENs are difficult to diagnose and treat because they can be hormonally active, ectopically secreting various biologically active substances that can cause specific syndromes requiring treatment and because 50-80\% of pNENs are malignant (except for insulinomas) and require treatment approaches that differ from those used for the most common adenocarcinomas (Ito et al. 2012). Approximately $60 \%$ of patients with pNENs develop liver metastases, while only $27 \%$ of all patients with gastroenteropancreatic NENs develop liver metastases; the lifelong incidence of liver metastases in patients with pNENs is as high as 85\% (Proye 2001, Fitzgerald et al. 2008, Lawrence et al. 2011). The heterogeneity of the clinical presentation of pNENs presents a great challenge for treatment and mechanism research.

Long non-coding RNAs (lncRNAs) are a kind of nonencoded RNA with a length of more than 200 nucleotides and little or no protein-coding function. However, it has been found that lncRNAs have important biological functions (Hickey et al. 2014, Yang et al. 2014, Bartoniceketal.2016). The abnormal expression of lncRNAs is closely related to human diseases, especially cancer, in which lncRNAs are mainly involved in tumor initiation, progression, metastasis and recurrence (Bartonicek et al. 2016). The $H 19$ gene is located on chromosome 11 in humans and is a maternally expressed imprinted gene that plays a vital role in mammalian development (Keniry et al. 2012). Increasing evidence has shown that H19 is upregulated in diverse cancer types, including bladder cancer, hepatocellular carcinoma, colon cancer, breast cancer and pancreatic cancer (Raveh et al. 2015). The overexpression of H19 in cancer patients highlights its tumorigenic properties, while the detailed molecular mechanisms remain poorly understood. However, due to the lack of H19 expression in Wilm's tumor, embryonic rhabdomyosarcoma and Beckwith-Wiedmann syndrome, H19 may function as a tumor suppressor in these tumors (Yoshimizu et al. 2008). In general, lncRNA H19 plays a carcinogenic role in most tumors, but in a few tumor types, lncRNA H19 plays a tumor-suppressive role. The specific expression and mechanism of lncRNA H19 in pNENs needs to be further elucidated.
$V G F$ is a highly conserved inducible gene for neurotrophic factors and was initially found in the induction and generation of pheochromocytoma cells treated with nerve growth factor (Salton et al. 1991, Rossi et al. 1992). VGF can be upregulated by many neurotrophic factors, including BDNF (brain-derived neurotrophic factor), NT-3 (neurotrophin-3) and 5-HT (serotonin) (Thakker-Varia \& Alder 2009). VGF produces a $68 \mathrm{kDa}$ polypeptide precursor (VGF precursor), which is induced and synthesized in large amounts and relatively selectively in neurons and neuroendocrine cells and that generates many shorter polypeptides through the posttranslational processing of neuroendocrinespecific prohormone convertases (Sibilia et al. 2010, Li et al. 2017). VGF proteins and polypeptides are widely distributed in the brain, especially in the hypothalamus and hippocampus. VGF is also widely distributed in peripheral tissues, including pituitary, adrenal, pancreatic and gastrointestinal myenteric plexus tissues and endocrine cells. VGF proteins are associated with multiple neuroendocrine functions, including the regulation of ingestion, energy balance, regeneration and homeostasis, synapse strengthening, long-term potentiation (LTP) and anti-depression (Sadahiro et al. 2015, Jiang et al. 2018).

In this study, with lncRNA microarrays, we identified a series of aberrantly expressed lncRNAs in pNENs. Subsequently, H19 was found to be upregulated in tumor tissues of pNENs with malignant behavior and was associated with poor survival. Then, mechanistic studies provided evidence that H19 promotes pNEN tumorigenesis and progression by directly binding to VGF. In addition, VGF was highly overexpressed in pNEN tissues and was positively correlated with H19 expression to promote cancer progression. Moreover, higher VGF expression was markedly related to poor differentiation and advanced stage. RNA sequence analysis revealed that $\mathrm{H} 19$ activates PI3K/Akt/CREB signaling pathways to promote pNEN progression by interacting with VGF.

\section{Materials and methods}

\section{Patient samples}

Tumors and their corresponding adjacent normal tissues as well as metastatic tissues were obtained from 39 patients who were diagnosed with nonfunctional pNENs and who underwent surgical resection at the Changzheng Hospital (Shanghai, China) between April 2014 and September 2017. The follow-up information of the 
39 patients was available for an average period of 45 months. We obtained the patients' written informed consent and approval from the Committee on Ethics of Biomedicine, Second Military Medical University prior to the use of the specimens for research purposes.

\section{QGP-1 cell line and cell culture}

The QGP-1 cell line was obtained from the Cobioer Company (Nanjing, China) and was grown in RPMI-1640 medium (Gibco, Thermo Fisher Scientific) supplemented with $10 \%$ fetal bovine serum (HyClone, Thermo Fisher Scientific) and 1\% penicillin/streptomycin (Life Technologies, Thermo Fisher Scientific). The QGP-1 cell line was authenticated with short tandem repeat (STR analysis).

\section{Primary tumor cell culture}

Due to the poor availability of other pNEN cell lines, we developed a culture method using primary cancer cells from human pNEN tumor samples in vitro. Mouse models have been developed to establish cultures from patientderived xenografts (PDXs). Briefly, fresh tumor tissue was mechanically sectioned into smaller fragments and implanted into immune-deficient mice. Then, when the xenografts grew to a certain size, they were resected to a size of $1.0 \mathrm{~cm}^{3}$ without necrosis and were immediately put in ice-cold RPMI-1640 supplemented with 1\% penicillin/ streptomycin. After removing the blood clots, the tumors were rinsed with sterile PBS and divided into small fragments. Then, the fragments were incubated with $0.2 \%$ collagenase, $0.01 \%$ hyaluronidase and $0.002 \%$ DNase I for $1 \mathrm{~h}$ at $37^{\circ} \mathrm{C}$. After passing through a $40 \mu \mathrm{m}$ mesh sieve, the cell suspension was washed twice and centrifuged at a speed of $300 \boldsymbol{g}$ for $20 \mathrm{~min}$. The cells were collected and incubated in dishes supplemented with RPMI-1640 containing $10 \% \mathrm{FBS}$ at $37^{\circ} \mathrm{C}$ with $5 \% \mathrm{CO}_{2}$. After $12 \mathrm{~h}$ of culture, the first medium change was performed. Function and mechanism experiments can be performed with these cells for two to three generations.

\section{IncRNA microarrays}

The lncRNA expression characteristics of pNENs were investigated by Arraystar Human lncRNA microarray V3.0, which contains lncRNAs collected from several databases such as the University of California Santa Cruz (UCSC), Ensembl and RefSeq databases, and the lncRNAs reported in the literature, were also included. The microarray and data collection were conducted by KangChen Biotech (Shanghai, China).

\section{Plasmid constructs and transfection}

The specific shRNA sequences for lncRNA H19 and VGF silencing were generated by Sangon Biotech (Shanghai, China) and inserted into the pLKO.1 vector (Addgene). The shRNA sequences are listed in Supplementary Table 1 (see section on supplementary data given at the end of this article). The human H19 and VGF overexpression pCMV3-H19 and pCMV3-VGF vectors were obtained from OBiO Technology (Shanghai, China). Then, the shRNA and overexpression lentiviruses were obtained according to the specifications of Lipofectamine 3000 (Life Technologies) and were transduced into QGP-1 cells or primary pNEN tumor cells. The transfection efficiencies were verified by RT-qPCR or Western blotting.

\section{MTT proliferation assay}

Cells were seeded into 96-well plates. MTT solution (Sigma-Aldrich) was added, and the crystals were dissolved in DMSO (Sigma-Aldrich). The color absorbance was recorded at $490 \mathrm{~nm}$ with a microplate reader (Novostar, Becton Dickinson).

\section{Colony formation assays}

Cells (500 cells/well) were cultured in $35 \mathrm{~mm}$ dishes for 2 weeks. After stopping the growth, cells were stained with Giemsa (Sigma-Aldrich).

\section{Migration and invasion assays}

Transwell chambers with $8 \mu \mathrm{m}$ pores without Matrigel bedding (Corning, 354578) were used to analyze cell migration, and chambers with Matrigel bedding (Corning, $356234)$ were used to analyze cell invasion. The migration and invasion abilities of the cells were measured according to the manufacturer's instructions.

\section{IncRNA transcription, RNA pulldown and mass spectrometry analyses}

The transcription of lncRNA H19 was conducted with a MEGAscript Kit (Thermo Fisher Scientific) in vitro. The full sequence of H19 was inserted into the pET-28a plasmid with a T7 RNA polymerase promoter site. The primers used for H19 PCR amplification are shown (c) 2019 Society for Endocrinology Published by Bioscientifica Ltd. Printed in Great Britain 
in Supplementary Table 2. Then, with the restriction enzyme, the linearized plasmid was used as a template for in vitro transcription following the manufacturer's instructions. The Pierce RNA 3' End Desthiobiotinylation Kit (Thermo Fisher Scientific) was used to cause the T4 RNA ligase to attach a single biotinylated nucleotide to the 3' terminus of IncRNA H19. RNA pulldown was performed using the MagNENic RNA-Protein Pull-Down kit (Thermo Fisher Scientific) in accordance with the manufacturer's instructions. The protein bands on the gel were stained with Coomassie brilliant blue. The bands of interest were identified by mass spectrometry (MS) and confirmed by Western blotting.

\section{RNA immunoprecipitation}

RNA immunoprecipitation (RIP) experiments were performed using a Magna RIP RNA-Binding Protein Immunoprecipitation Kit (Millipore) according to the manufacturer's instructions.

\section{RNA isolation and quantitative RT-qPCR}

RNA used for the real-time PCR experiments was extracted with TRIzol reagent (Invitrogen). Real-time PCR was performed using an ABI VIIA7 system with SYBR Premix Ex Taq (TaKaRa). Gene expression was normalized to that of the housekeeping gene using the $2^{-\Delta \Delta C T}$ method. The primers are shown in Supplementary Table 2.

\section{Western blotting}

Total cell lysates were prepared using Pierce IP Lysis Buffer supplemented with protease and phosphatase inhibitors (Thermo Scientific) and were quantified with the BCA Protein Assay Kit (Thermo Scientific). The protein samples were separated via SDS-PAGE, transferred onto nitrocellulose membranes and were finally developed using the enhanced chemiluminescence (ECL) substrate (Thermo Scientific). The following antibodies were used: VGF (Abcam), PI3K, P-PI3K, AKT, P-AKT, P-CREB, CREB, CBP and GAPDH (Cell Signaling Technology).

\section{QGP-1 xenograft model}

All in vivo studies were approved by the Committee on Ethics of Shanghai Institute of Materia Medica and conducted in compliance with the Institutional Animal Care and Use Committee guidelines of Shanghai Institute of Materia Medica. Nude mice were inoculated with different kinds of QGP-1 cells $\left(1 \times 10^{7} /\right.$ mice $)$. After tumorigenesis, the increase in tumor volume was recorded using digital calipers, and tumor volumes were estimated using the formula $\mathrm{L} \times \mathrm{W}^{2} / 2$. The body weight during the course of the study was also monitored. At the end of the studies, the mice were killed and the tumors were extracted and weighed.

\section{Next-generation RNA sequencing (RNA-seq)}

RNA-seq of H19- or VGF-knockdown cells was carried out and analyzed on the free online platform of the Majorbio I-Sanger Cloud Platform (Majorbio Biopharm Technology, China). Gene set enrichment analysis (GSEA) was carried out using GSEA v3.0. GSEA and the gene set collection of the KEGG pathways were used for enrichment analysis.

\section{Statistical analysis}

Data are expressed as the mean \pm standard deviation from at least three replicates. Comparisons between groups were performed using the two-tailed Student's $t$ test or one- or two-way ANOVAs. The results were considered to be statistically significant when $P \leq 0.05$.

\section{Results}

\section{IncRNA expression profiles in pNEN tissue}

To identify the eligible lncRNAs in pNENs, we performed gene expression array analysis on five paired tumor tissue and adjacent tumor tissue samples of patients with pNENs with G2 grading who received a curative operation as a primary treatment in the Changzheng Hospital. In total, we identified and selected the top 300 lncRNA transcripts that were significantly differentially expressed (fold change $>2$ or $<0.5, P \leq 0.05$ ) (Fig. 1 A). Of all of the 300 abnormally expressed lncRNAs, we noticed that H19 was downregulated in pNEN tissues. H19 is increasingly described as playing key roles in the progression and metastasis of cancers from different tissue origins. We wanted to further investigate the expression and underlying mechanisms of H19 in pNENs. Therefore, the H19 expression in 39 paired samples of pNEN tissues and corresponding adjacent noncancerous tissues was assessed by qPCR. We also examined the expression of H19 in liver metastatic lesions. According to clinical data, malignant tumor behavior is defined as local invasion, positive regional lymph node metastasis, microvascular tumor 
B

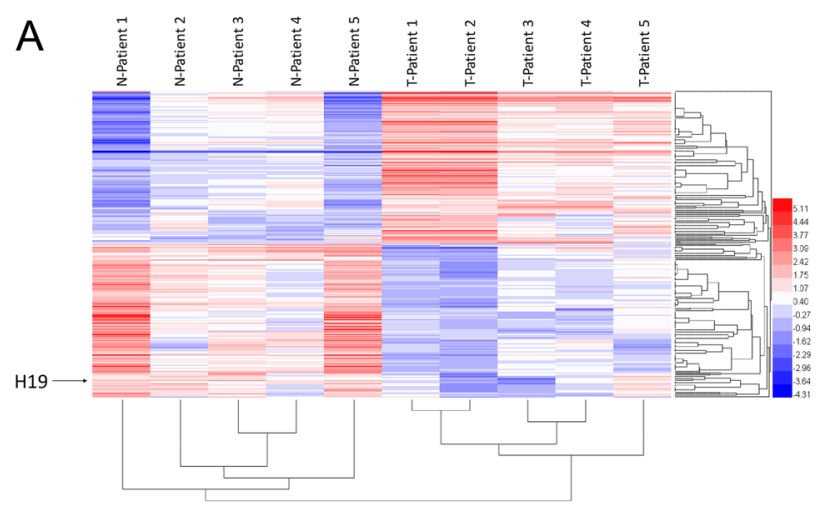

C

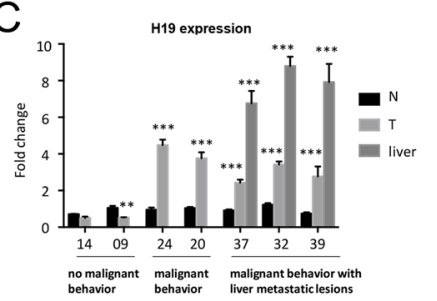

H19

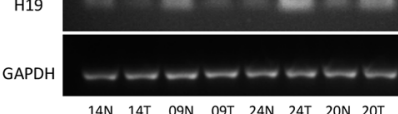

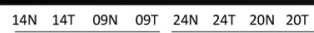
no malignant behavior malignant behavior

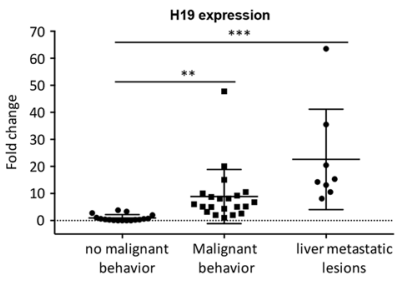

D

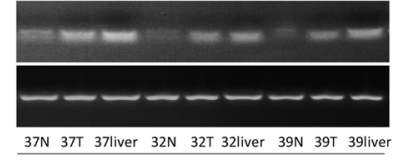

malignant behavior with
E

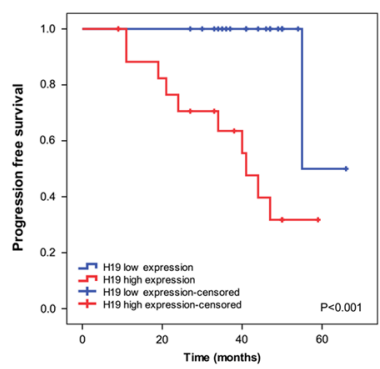

F

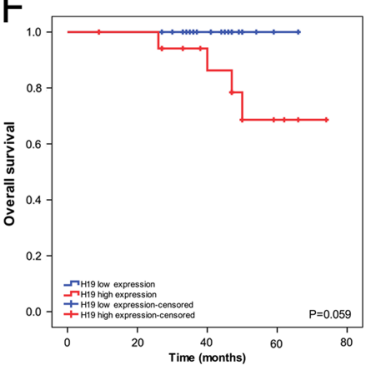

Figure 1

High expression of $\mathrm{H} 19$ is correlated with poor prognosis in pNEN patients. (A) Heatmap of differentially expressed IncRNAs in 5 pNEN tumor tissue and paired nontumor tissue samples. T, tumor tissues; N, nontumor tissues. (B) Relative H19 expression in tissues $(n=39)$ identified by qPCR. (C and D) Relative $\mathrm{H} 19$ expression in pNEN tissues with no malignant behavior, malignant behavior and liver metastasis using PCR and qPCR assays. 14, 09, 24, 20, 37, 32 and 39 represent the patient number; N represents the adjacent tissues; T represents the tumor tissues, 37liver, 32liver and 39liver correspond to the patient's liver metastatic tumor tissue. (E) Kaplan-Meier survival analysis demonstrated that patients with high $\mathrm{H} 19$ expression had poor progression-free survival (PFS) compared to the PFS of those with low $\mathrm{H} 19$ expression. (F) OS curve of the high-level and low-level groups divided by $\mathrm{H} 19$ expression. ( ${ }^{*} P \leq 0.05,{ }^{*} P \leq 0.01,{ }^{*} * P \leq 0.001$.) A full colour version of this figure is available at https://doi.org/10.1530/ERC-18-0552.

thrombosis or liver metastasis. A tumor with no malignant behavior does not exhibit any of the abovementioned features. However, to our surprise, the results showed that the expression of $\mathrm{H} 19$ was not uniform in pNENs, and there were samples with notably high H19 expression. After further analysis, we found that H19 showed low expression levels in pNENs with no malignant behavior, while in pNENs with malignant behavior, the expression of H19 was upregulated; in addition, H19 expression was significantly increased in liver metastatic lesions (Fig. 1B, C and D). Coincidentally, the samples from the five patients that we used for gene expression array all came from progression-free pNENs, so the level of H19 expression was downregulated in the heatmap.

\section{High expression of $\mathrm{H} 19$ is correlated with poor prognosis in pNEN patients}

By analyzing clinicopathological factors, we found that high H19 expression was correlated with malignant behavior in pNEN patients. We divided the samples into high (fold change $\geq 3, n=19$ ) and low (fold change $<3, n=20$ )
H19 expression groups for analysis. The analysis revealed that high expression of H19 was significantly correlated with tumor size $(P=0.017)$, lymph node metastasis $(P=0.044)$, local invasion $(P=0.044)$, synchronous liver metastasis $(P=0.001)$, tumor grading $(P=0.022)$, tumor TNM stage $(P<0.001)$, tumor progression $(P=0.001)$ and tumor-related death $(P=0.047)$ (Table 1$)$. Kaplan-Meier survival analysis demonstrated that the patients with high H19 expression had poor progression-free survival (PFS) compared to the PFS of those with low H19 expression $(P<0.001)$ (Fig. 1E). Moreover, the patients with high H19 expression had a poorer overall survival (OS) than those with low H19 expression, but there was no significant difference $(P=0.059)$ (Fig. $1 F)$.

\section{H19 knockdown inhibits cell proliferation, migration and invasion in pNENs}

To define the effect of H19 on pNEN development, we analyzed the endogenous expression of H19 in pNENs. Due to the poor availability of other pNEN cell lines, we developed a culture method using primary tumor cells 
Table 1 Correlation of H19 expression in pNEN with clinicopathologic features.

\begin{tabular}{|c|c|}
\hline Clinicopathologic features & H19 low expression $(n=20)$ \\
\hline Age & $48.8 \pm 10.8$ \\
\hline Female & 13 \\
\hline Symptoms & 8 \\
\hline \multicolumn{2}{|l|}{ Location } \\
\hline Head & 8 \\
\hline Body and tail & 12 \\
\hline Functional & 3 \\
\hline Size $(\mathrm{cm})$ & $3.5 \pm 2.0$ \\
\hline Lymph node metastasis & 1 \\
\hline Local invasion & 1 \\
\hline Microvascular invasion & 2 \\
\hline Synchronous liver metastasis & 0 \\
\hline Ki67 (\%) & $3.6 \pm 2.7$ \\
\hline \multicolumn{2}{|l|}{ Grading } \\
\hline G1 & 9 \\
\hline $\mathrm{G} 2$ & 11 \\
\hline G3 & 0 \\
\hline \multicolumn{2}{|l|}{$\mathrm{T}$} \\
\hline $\mathrm{T} 1$ & 3 \\
\hline $\mathrm{T} 2$ & 11 \\
\hline T3 & 6 \\
\hline $\mathrm{T} 4$ & 0 \\
\hline \multicolumn{2}{|l|}{$\mathrm{N}$} \\
\hline NO & 19 \\
\hline N1 & 1 \\
\hline \multicolumn{2}{|l|}{$\mathrm{M}$} \\
\hline MO & 20 \\
\hline M1 & 0 \\
\hline \multicolumn{2}{|l|}{ TNM stage } \\
\hline I & 3 \\
\hline II & 16 \\
\hline III & 1 \\
\hline IV & 0 \\
\hline Tumor progression & 1 \\
\hline Tumor-related death & 0 \\
\hline
\end{tabular}

\begin{tabular}{|c|c|}
\hline H19 high expression $(n=19)$ & P value \\
\hline $56.3 \pm 9.8$ & $0.029 *$ \\
\hline 11 & 0.648 \\
\hline \multirow[t]{2}{*}{14} & $0.034 *$ \\
\hline & 0.584 \\
\hline \multicolumn{2}{|l|}{6} \\
\hline \multicolumn{2}{|l|}{13} \\
\hline 1 & 0.605 \\
\hline $5.5 \pm 2.8$ & $0.017 *$ \\
\hline 6 & $0.044^{*}$ \\
\hline 6 & $0.044 *$ \\
\hline 2 & 1.000 \\
\hline 8 & 0.001 * \\
\hline \multirow{2}{*}{$9.2 \pm 8.0$} & $0.008 *$ \\
\hline & $0.022 *$ \\
\hline \multicolumn{2}{|l|}{2} \\
\hline \multicolumn{2}{|l|}{15} \\
\hline \multicolumn{2}{|l|}{2} \\
\hline \multicolumn{2}{|r|}{$0.017^{*}$} \\
\hline \multicolumn{2}{|l|}{0} \\
\hline \multicolumn{2}{|l|}{6} \\
\hline \multicolumn{2}{|l|}{8} \\
\hline \multicolumn{2}{|l|}{5} \\
\hline & $0.044 *$ \\
\hline \multicolumn{2}{|l|}{13} \\
\hline \multicolumn{2}{|l|}{6} \\
\hline & $0.001 *$ \\
\hline \multicolumn{2}{|l|}{11} \\
\hline \multicolumn{2}{|l|}{0} \\
\hline & $<0.001 *$ \\
\hline \multicolumn{2}{|l|}{0} \\
\hline \multicolumn{2}{|l|}{6} \\
\hline \multicolumn{2}{|l|}{5} \\
\hline \multicolumn{2}{|l|}{8} \\
\hline 10 & $0.001 *$ \\
\hline 4 & $0.047 *$ \\
\hline
\end{tabular}

$\mathrm{H} 19$ low expression (fold change $<3$ ); $\mathrm{H} 19$ high expression (fold change $\geq 3$ ). $\star P \leq 0.05, * * P \leq 0.01, * * * P \leq 0.001$

from human pNEN tumor samples in vitro. In addition, the QGP-1 cell line was used in the research. QGP-1 cells are derived from pNEN tumors with well-circumscribed nodular lesions in the tail of the pancreas and vascular invasion and metastases of tumor cells to the peripancreatic lymph nodes and the liver (Kaku et al. 1980). First, we examined the baseline lncRNA H19 levels in eight cell lines $(24 \mathrm{~N}, 20 \mathrm{~N}, 37 \mathrm{~N}, 24 \mathrm{~T}, 20 \mathrm{~T}, 37 \mathrm{~T}$, QGP-1 and 37 liver) by qPCR (Fig. 2A). We selected QGP-1, 37T and 24T cells for the following experiments. Next, QGP-1, 37T and 24T cells were transfected with the H19 shRNA lentiviruses and the control lentiviruses. The knockdown efficiencies were verified by qPCR (Fig. 2B). MTT assays were performed and showed that H19 knockdown significantly decreased the proliferation abilities of QGP-1 cells compared to the proliferation abilities of the respective controls (Fig. 2C).
This result was also confirmed in pNEN primary tumor cells (Fig. 2D and E). In addition, colony formation assays showed that H19 knockdown inhibited colony formation (Fig. 2F). We noticed that H19-knockdown QGP-1 cells grew more slowly than QGP-1 control cells and had a different morphology (Fig. 2G). The H19-knockdown QGP-1 cells exhibited shrinkage and restricted growth, whereas the control QGP-1 cells exhibited an ellipse shape and ovoid morphology and spread and grew well. These findings indicated that H19 may contribute to the invasion and migration of pNEN cells. Therefore, these cells were assessed using Transwell assays. Furthermore, Transwell assays showed that the invasiveness and migration capabilities of the QGP-1 and 37T cells were significantly reduced when $\mathrm{H} 19$ expression was decreased (Fig. 2H). Taken together, the above results indicate 

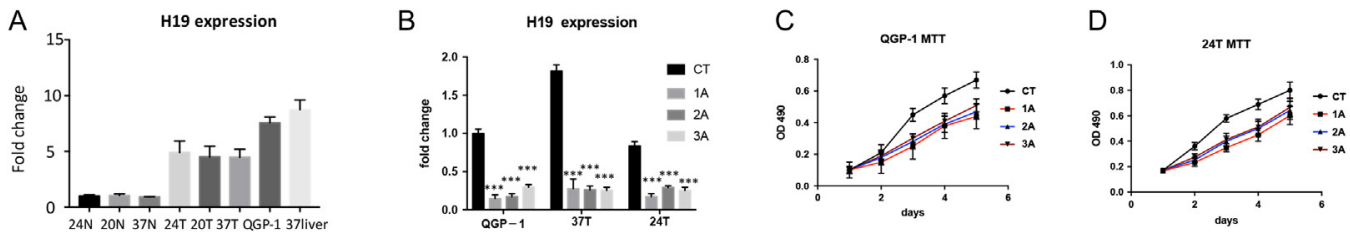

E
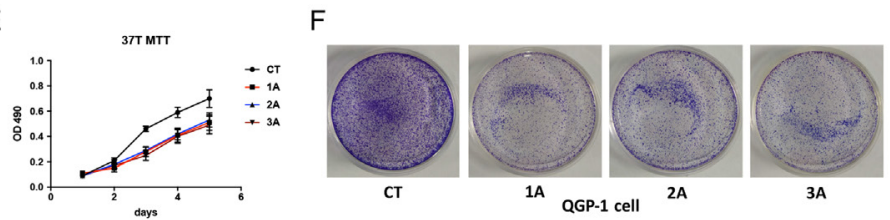

G

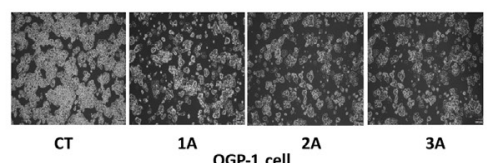

$\mathrm{H}$

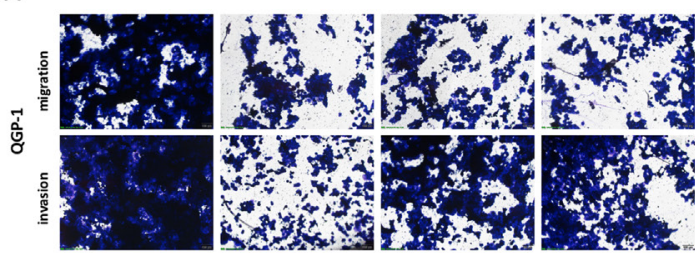

I J
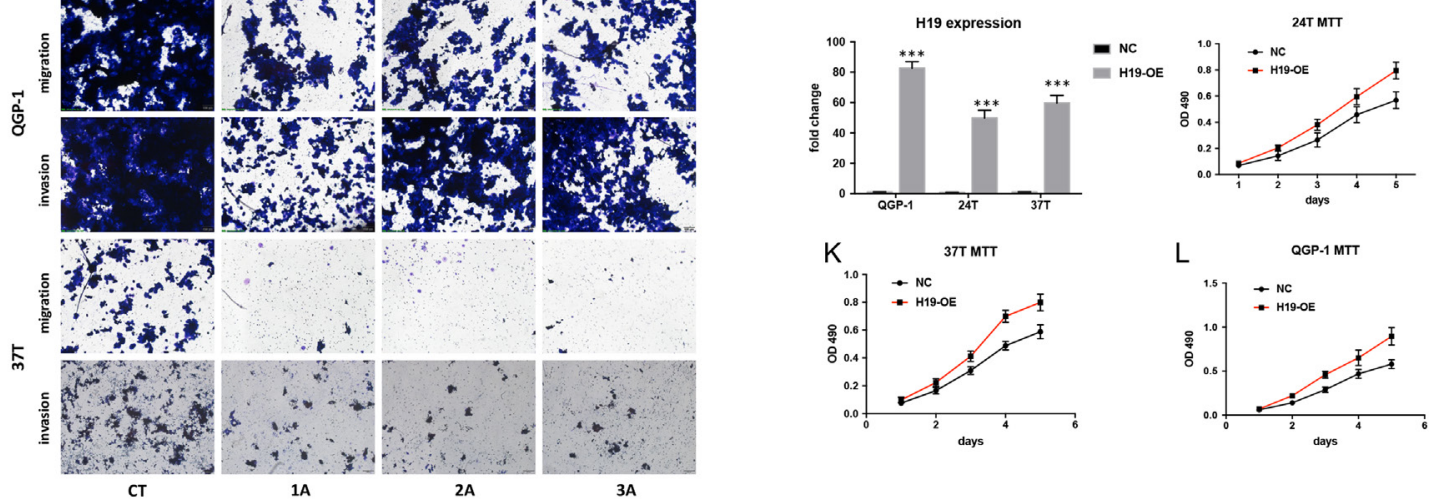

M

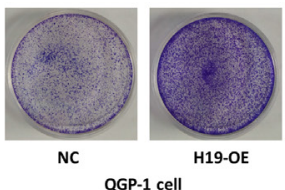

$\mathrm{N}$
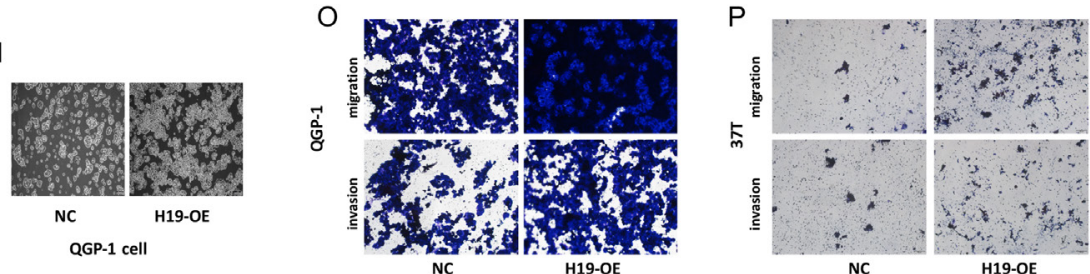

Figure 2

H19 promotes cell proliferation, migration and invasion in pNENs. (A) Baseline IncRNA H19 levels in eight cell lines (24N, 20N, 37N, 24T, 20T, 37T, QGP-1 and 37 liver) identified by qPCR assay. (B) The $\mathrm{H} 19$ knockdown efficiencies were verified by qPCR assay. CT represents cell transfection with control lentivirus; 1A, 2A and 3A represent cells transfected with three kinds of H19 shRNA lentiviruses. (C, D and E) Proliferation of H19-knockdown and control QGP-1 and pNEN primary tumor cells. (F) Colony formation assays of H19-knockdown and control QGP-1 cells. (G) Cell morphology of H19-knockdown and control QGP-1 cells. (H) Transwell assays showed that the invasiveness and migration capabilities of QGP-1 cells and 37T cells were significantly reduced when $\mathrm{H} 19$ expression was decreased. (I) The H19 overexpression efficiencies were verified by qPCR assay. NC represents cells transfected with control lentivirus; $\mathrm{H} 19-\mathrm{OE}$ represents cells transfected with $\mathrm{H} 19$ overexpression lentivirus. (, K and L) Proliferation of H19-overexpressing and control QGP-1 cells and pNEN primary tumor cells. (M) Colony formation assays of H19-overexpressing and control QGP-1 cells. (N) Cell morphology of H19-overexpressing and control QGP-1 cells. (O and P) Transwell assays showed that the invasiveness and migration capabilities of QGP-1 cells and 37T cells were significantly increased when $\mathrm{H} 19$ was overexpressed. ( $* P \leq 0.05, * \star P \leq 0.01, * * * P \leq 0.001$.) A full colour version of this figure is available at https://doi.org/10.1530/ERC-18-0552.

that H19 plays a key role in regulating the malignant behaviors of pNENs.

\section{H19 overexpression promoted pNEN cell proliferation, migration and invasion}

To further confirm the function of H19 in pNENs, we overexpressed H19 in QGP-1, 01T and 37T cells, which was verified with a qPCR assay (Fig. 2I). Overexpression of $\mathrm{H} 19$ promoted cell proliferation as shown by the MTT assays (Fig. 2H, K and L). In addition, H19 overexpression promoted colony formation of QGP-1 cells (Fig. 2M). Moreover, the cell morphology changed when H19 was overexpressed in QGP-1 cells (Fig. 2N). These phenotypic modifications are sometimes reported when epithelial-to-mesenchymal 
transition (EMT) occurs. Additionally, more pNEN cells migrated and invaded after the ectopic expression of H19 in QGP-1 and 37T cells (Fig. 20 and P).

\section{H19 promotes pNEN tumor growth in vivo}

The above in vitro data indicated that lncRNA H19 promoted pNEN cell proliferation, migration and invasion. Furthermore, xenograft mouse models were applied to detect the tumorigenic role of H19. QGP-1 cells were transfected with control lentivirus and H19 shRNA lentivirus (1A, 2A, 3A). The transfected QGP-1 cells were subcutaneously implanted into 5-week-old nude mice. We then measured the tumor volumes and body weights at the indicated time points (Fig. 3C and D). After several weeks of xenograft growth in vivo, we killed the mice, removed the tumors and assessed the tumor weights. We compared the sizes of the xenograft tumors among the groups and found that the H19knockdown group exhibited a dramatic decrease in tumor volume and weight (Fig. 3A, B and E). In addition, H19-overexpressing QGP-1 cells and control cells were injected into nude mice. Similarly, tumor volumes and body weights were measured at the indicated time points (Fig. 3H and I). Then, the mice were killed, and the tumor weights were measured after several weeks of growth, and we found that the H19 overexpression group showed a significant increase in tumor weight and size compared to the same parameters in the control group (Fig. 3F, G and J). Furthermore, more Ki67+ cells were found in the control tumor tissues than in the H19-knockdown tissues, whereas we detected stronger Ki67 expression in tumors derived from the H19-overexpression groups than in those derived from the control groups (Fig. 3K).
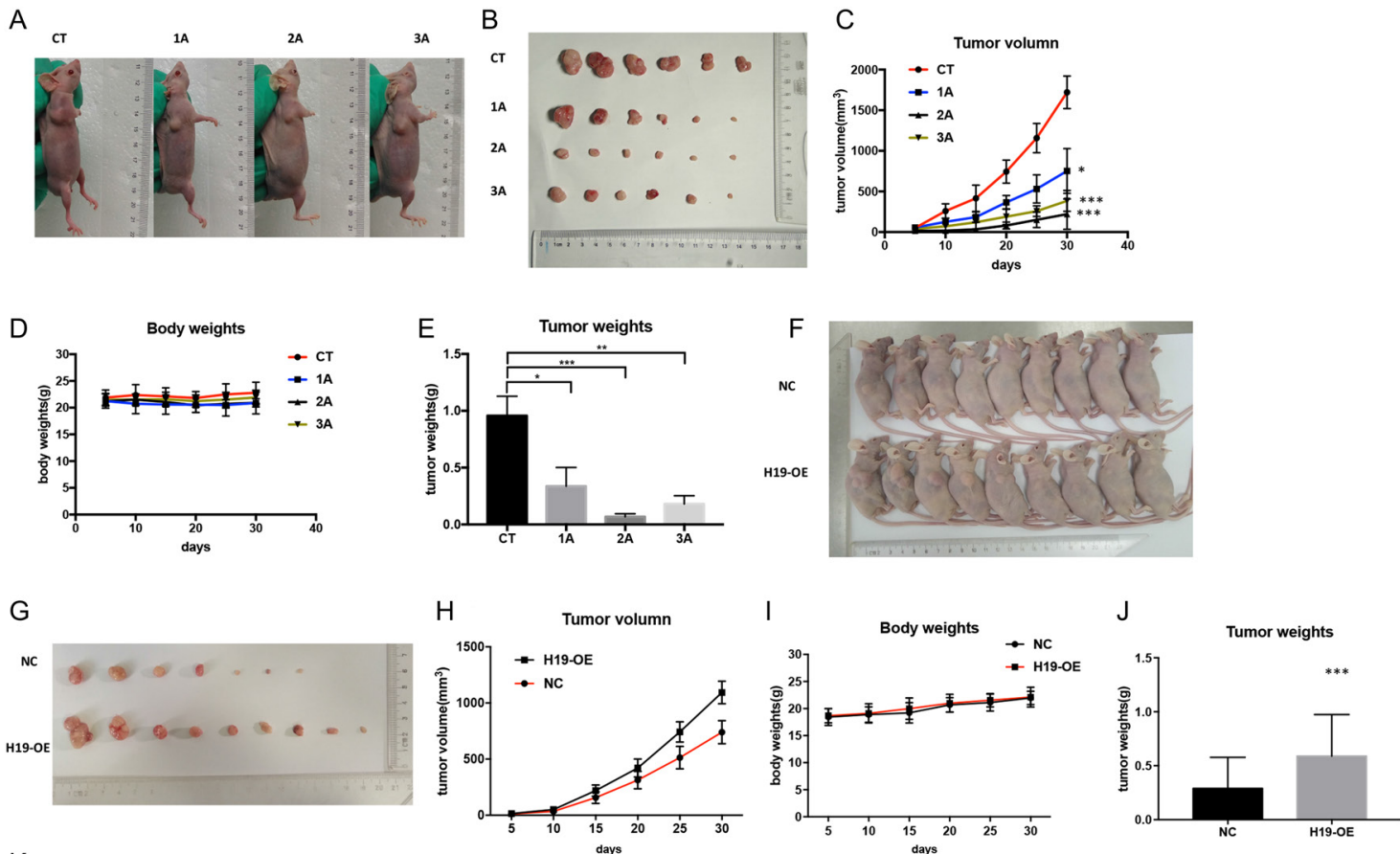

$\mathrm{K}$

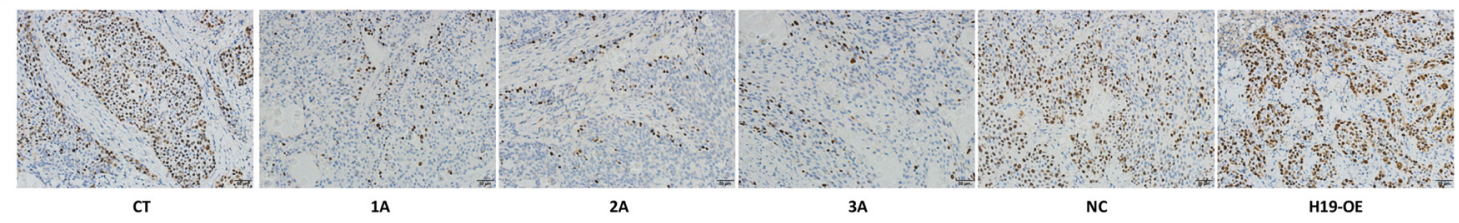

Figure 3

H19 promotes pNEN tumor growth in vivo. (A, B, F and G) Representative images of tumors formed in nude mice injected subcutaneously with QGP-1 cells are shown. (A and B: H19 knockdown and control. F and G: H19 overexpression and control). (C and H) The tumor volume was periodically measured for each mouse, and the tumor growth curve was plotted. (D and I) The body weights were periodically measured for each mouse, and the body weight curve was plotted. (E and J) The tumor weights were measured when the tumors were harvested. (K) Representative images ( $\times 200)$ of IHC staining of Ki67 in the tumors. ( ${ }^{2} P \leq 0.05, * \star P \leq 0.01, * \star * P \leq 0.001$.) A full colour version of this figure is available at https://doi.org/10.1530/ERC-18-0552. 


\section{IncRNA H19 interacts with VGF and regulates VGF expression in pNENs}

A previous study demonstrated that lncRNAs can interact with proteins to regulate gene expression. Papers have reported that IncRNA H19 can interact with Ago2 in gallbladder cancer (Wang et al. 2016) and with P53 in gastric cancer (Yang et al. 2012). Hence, we performed RNA pulldown and mass spectra (MS) analyses to identify potential interacting proteins. After H19 transcription, biotin-labeling and RNA pulldown, the retrieved proteins were subjected to SDS-PAGE analysis. The differential band that appeared in the H19 lane was cut for identification by MS (Fig. 4A). The MS results revealed that VGF, a secreted protein and neuropeptide precursor, was the protein with the highest spectral counts binding to biotin-labeled H19. Western blotting further confirmed the significant enrichment of VGF with lncRNA H19
(Fig. 4B). Moreover, RNA immunoprecipitation was performed with a VGF antibody from nuclear extracts of QGP-1 cells. We found significant enrichment of H19 with VGF antibody compared with that of the IgG control antibody by DNA gel electrophoresis, PCR and qPCR (Fig. 4C and D). Collectively, these results demonstrated a tight interaction between lncRNA H19 and VGF.

To determine whether H19 regulates VGF expression, qPCR and Western blotting were conducted to detect VGF expression in pNEN cells with H19 knockdown or overexpression. We found that the expression of VGF was significantly downregulated after H19 was knocked down and that VGF was upregulated after H19 was overexpressed in QGP-1 cells and primary pNEN cancer cells (Fig. 4E, F, G and $\mathrm{H}$ ). Moreover, the IHC results showed a similar trend in the QGP-1 xenograft model samples (Fig. 4I). Taken together, these results showed that lncRNA H19 interacts with VGF and regulates VGF expression in pNENs.
A
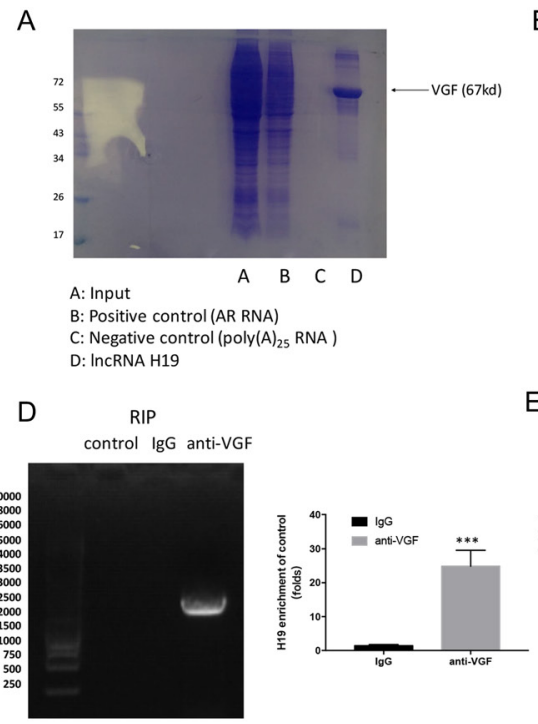

B

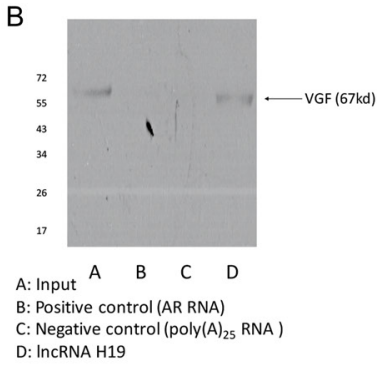

E
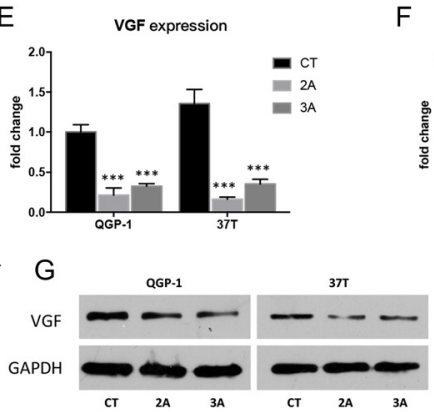

C

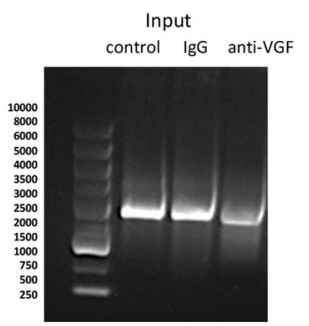

$\mathrm{F}$
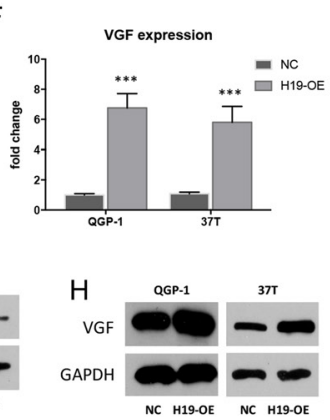

I
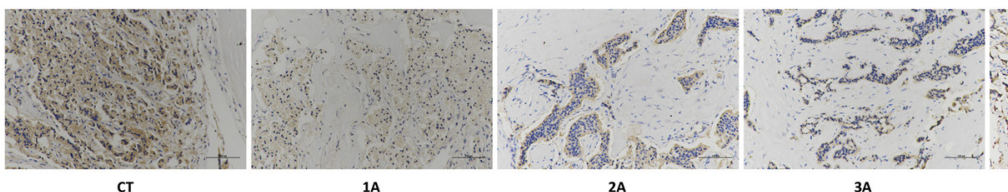

\section{Figure 4}

IncRNA H19 interacts with VGF and regulates VGF expression in pNENs. (A) Proteins retrieved from the H19-pulldown assay were analyzed by SDS-PAGE. (B) Western blotting confirmed the significant enrichment of VGF with IncRNA H19 using an anti-VGF antibody. (C and D) RIP assay was performed in QGP-1 cells to determine the binding condition between IncRNA H19 and VGF. (E, F, G and H) Assessing VGF expression in H19-knockdown and H19-overexpressing QGP-1 cells with Western blotting and qPCR assays. (I) Representative images $(\times 200)$ of IHC staining of VGF in H19-knockdown or H19-overexpressing xenograft tumors. ( ${ }^{*} P \leq 0.05, * * P \leq 0.01, * * * P \leq 0.001$.) A full colour version of this figure is available at https://doi.org/10.1530/ ERC-18-0552. 


\section{Elevated VGF is correlated with pNEN aggressiveness}

Based on the correlation between H19 and VGF, we wanted to validate whether VGF was differentially expressed in pNEN tissues. The VGF expression in 39 paired pNEN tissues was measured by IHC, qPCR and Western blotting. As the results showed, VGF was upregulated in tumor tissues and was significantly upregulated in liver metastases (Fig. 5A, B and C). Additionally, assessing the clinical significance, we evaluated the correlation of VGF expression with clinicopathological characteristics, as shown in Table 2. We divided samples into high (fold change $\geq 10, n=21$ ) and low (fold change $<10, n=18$ ) VGF expression groups for analysis. The analysis revealed that the high expression of VGF was significantly correlated with lymph node metastasis $(P=0.010)$, local invasion $(P=0.010)$, synchronous liver metastasis $(P=0.004)$, tumor grading $(P=0.011)$, tumor TNM stage $(P<0.001)$ and tumor progression $(P<0.001)$. These analyses also showed that VGF was more highly expressed in tumors with malignant behavior than in progression-free pNENs. More importantly, higher VGF expression was observed in patients with metastasis than in those without metastasis. Taken together, these results indicate that VGF has important functions in pNEN tumorigenesis. These results are all consistent with the observed increased H19 expression in pNEN tissues with malignant behavior, indicating that VGF is likely a target of H19. The correlation between $\mathrm{H} 19$ and VGF expression was further analyzed in pNEN tissues, and a positive correlation was found, which further supports our hypothesis (Fig. 5D). Furthermore, Kaplan-Meier survival analysis demonstrated that patients with high VGF expression had poor PFS compared to the PFS of those with low VGF expression $(P<0.001)$ (Fig. 5E). Moreover, the patients with high VGF expression had a poorer OS than those with low VGF expression, but there was no significant difference $(P=0.067)$ (Fig. 5F).

\section{VGF promotes pNEN cells proliferation, migration and invasion}

Because our results showed that H19 promoted pNEN cell proliferation, migration and invasion, we next examined the functional role of VGF in pNEN progression. VGF knockdown and overexpression lentiviruses were obtained. After transfection, the knockdown and overexpression efficiencies were verified by qPCR and Western blotting (Fig. 5G and H). The MTT assay showed that VGF overexpression or knockdown promoted or delayed cell proliferation, respectively, compared with the findings of the respective control in QGP-1 cells and 37T cells (Fig. 5I and J). In addition, in vitro migration and invasion assays demonstrated that VGF overexpression or knockdown resulted in higher or lower rates of QGP-1 cell migration and invasion, respectively, compared with the migration and invasion rates of the controls (Fig. 5K). These findings provide evidence that VGF plays a similar role as H19 in pNEN progression. In addition, we performed rescue experiments to investigate whether $\mathrm{H} 9$ promoted pNEN progression via VGF. We overexpressed VGF in H19-knockdown pNEN cells and discovered that overexpression of VGF partially attenuated the decreased cell proliferation, migration and invasion capacity caused by H19 knockdown (Fig. 5L and M). Therefore, our findings indicated that VGF was a major downstream mediator of H19-induced tumorigenic and metastatic activity.

\section{Transcriptomic signature of pNENs with $\mathrm{H} 19$ or VGF knockdown}

To determine how H19 and VGF regulate cancer cell growth and metastasis, we performed RNA-seq analysis on three kinds of QGP-1 cells (control cells, H19-knockdown cells and VGF-knockdown cells) to identify the genomewide genes and pathways regulated by H19 or VGF. The comprehensive analysis of differential genes and associated signaling pathways after knockdown of H19 and VGF could further validate the relationship between H19 and VGF.

A heatmap of the differential genes that were modulated by more than two-fold in treated cells compared with their expression in the control cells was generated from the RNA-seq data and showed clustering of H19knockdown cells, VGF-knockdown cells and control cells (Fig. 6A). Genes with similar expression patterns usually have functional correlations, which reveals the potential similarity in transcriptional regulation between H19knockdown cells and VGF-knockdown cells. Furthermore, in the volcano plot, the upregulated and downregulated genes were also comparable in the H19-knockdown cells and the VGF-knockdown cells (Supplementary Fig. 1A and B).

Through transcriptome sequencing and analysis, we compared the H19-knockdown groups with the control groups, and the genes that were significantly altered ( $P \leq 0.05$, fold change $\geq 2$ ) with multiple correction were chosen as the H19-knockdown differential gene set. The same method was used to select the VGF-knockdown differential gene set. Gene ontology (GO) enrichment analysis of the two differentially expressed gene sets (c) 2019 Society for Endocrinology Published by Bioscientifica Ltd. Printed in Great Britain 


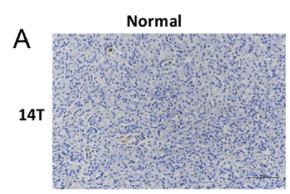

Normal
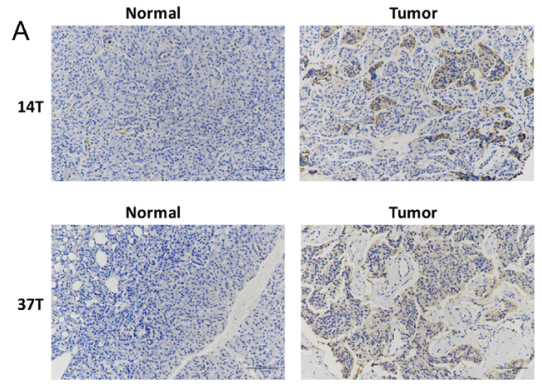

Tumor

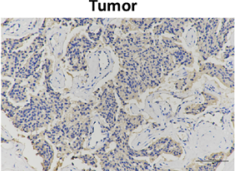

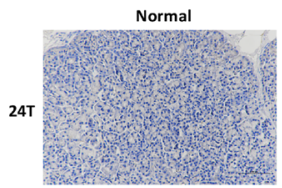

Liver

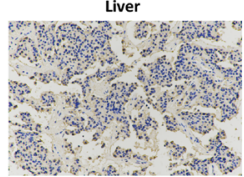

C var

VGF

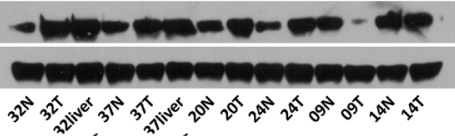

E
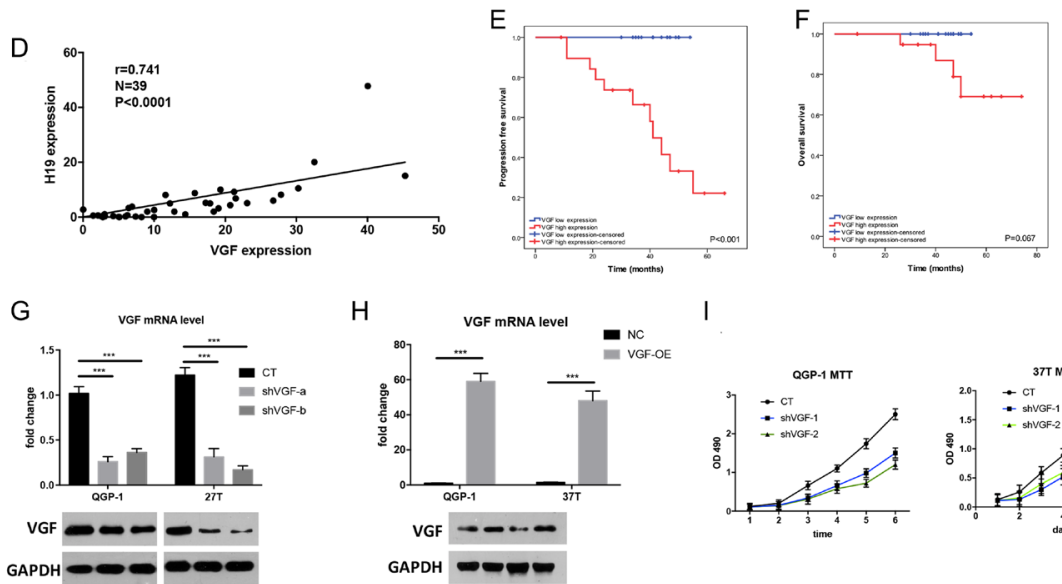

$\mathrm{H}$
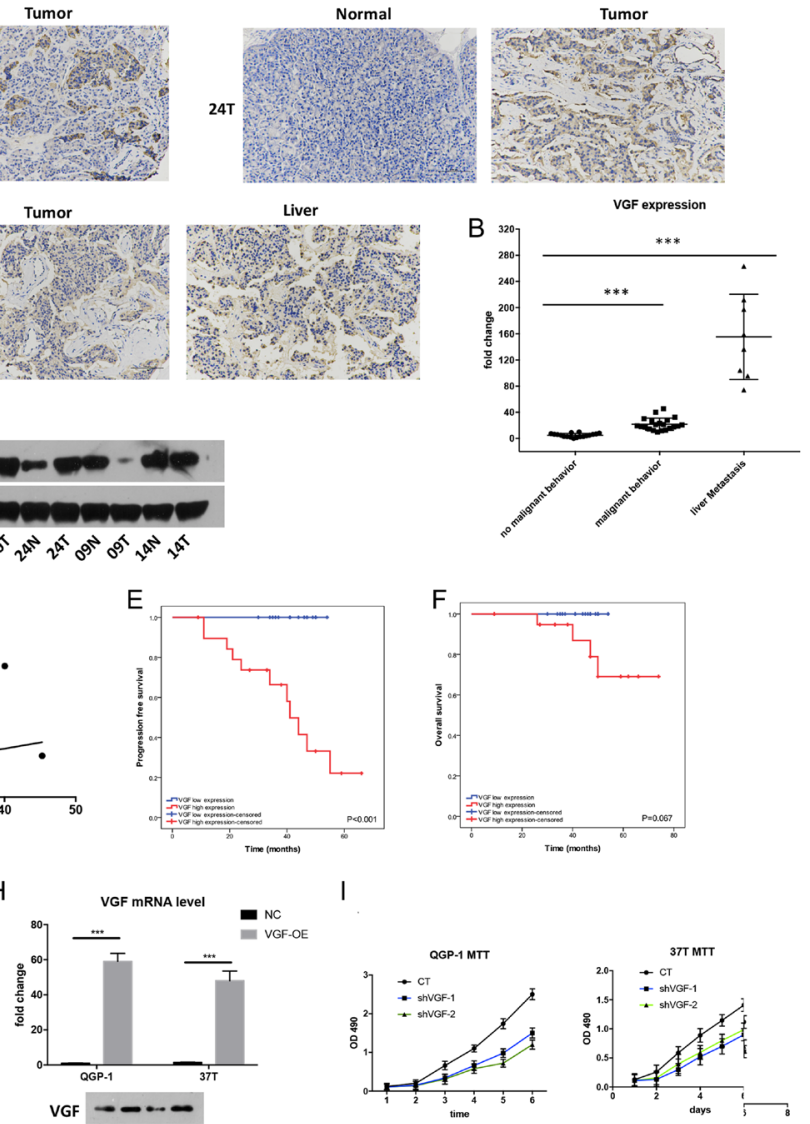

APDH
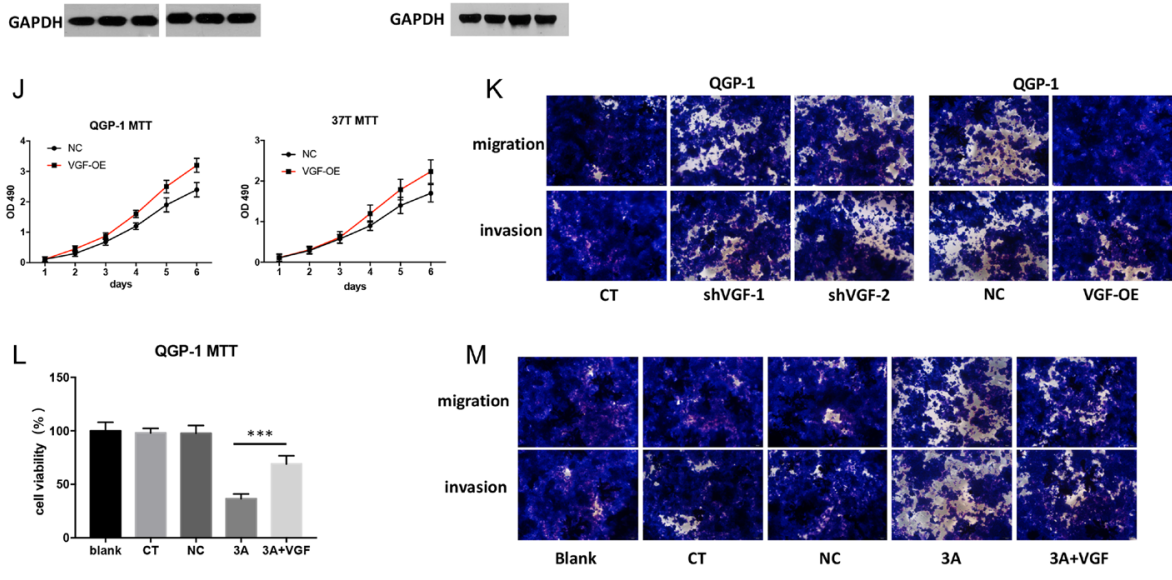

Figure 5

VGF, which promotes pNEN cell proliferation, migration and invasion, is elevated in pNENs and correlated with pNEN aggressiveness. (A, B and C) VGF expression in 39 paired pNEN tissues was measured by IHC, qPCR and Western blotting. (D) Pearson's correlation analysis showed a positive correlation between the expression levels of H19 and VGF in pNEN tissues ( $r=0.741, P<0.0001, n=39)$. (E) Kaplan-Meier survival analysis demonstrated that patients with high VGF expression had poor progression-free survival (PFS) compared to the PFS of those with low VGF expression. (F) Overall survival curve of the high-level and low-level groups divided by VGF expression. (G and H) The VGF knockdown and overexpression efficiencies were verified by qPCR and Western blotting assays. CT represents cells transfected with control lentivirus; shVGF-a and shVGF-b represent cells transfected with two kinds of VGF shRNA lentivirus. NC represents cells transfected with control lentivirus; VGF-OE represents cells transfected with VGF overexpression lentivirus. (I and J) Cell proliferation of VGF-knockdown, VGF-overexpressing and the corresponding control QGP-1 cells and pNEN primary tumor cells. (K) Transwell assays showed that the invasiveness and migration capability of QGP-1 cells were significantly changed when H19 was knocked down or overexpressed. (L) Overexpression of VGF partially attenuated the decreased cell proliferation caused by H19 knockdown in QGP-1 cells. Blank represents QGP-1 cells with no treatment; CT represents QGP-1 cells transfected with shRNA control lentivirus; NC represents QGP-1 cells transfected with overexpression control lentivirus; 3A represents QGP-1 cells transfected with $\mathrm{H} 19$ shRNA-3A lentivirus; 3A + VGF represents QGP-1 cells transfected with $\mathrm{H} 19$ shRNA-3A lentivirus and VGF overexpression lentivirus. (M) Overexpression of VGF partially attenuated the decreased cell migration and invasion capacity caused by $\mathrm{H} 19$ knockdown in QGP-1 cells. ( $* P \leq 0.05, * \star P \leq 0.01, * \star \star P \leq 0.001$.) A full colour version of this figure is available at https://doi.org/10.1530/ERC-18-0552. 
showed that the changes in biological process, cellular components and molecular functions were consistent (Supplementary Fig. 2). Thus, H19 and VGF regulate cancer cell growth and metastasis through the same signaling pathway. Interestingly, the results from the two gene set enrichment analyses derived from the KEGG pathway database indicated that most of the differentially expressed genes were involved in the PI3K-AKT signaling pathway in both differential gene sets (Fig. 6B). Therefore, we suspected that H19 targeted and regulated VGF expression mainly through the PI3K-AKT signaling pathway, exerting a role in promoting pNEN development. In addition, we analyzed the proteinprotein interaction (PPI) network between the first
300 genes of the two differential gene sets, and the results showed that the CBP (also named CREBBP) protein was at the center of the two PPI networks (Supplementary Fig. 3A and B). CBP is a CREB-binding protein (Chen \& Korenberg 1995) that is involved in the transcriptional coactivation of many different transcription factors. In addition, previous studies have shown that CREB is also an important component in the PI3K-AKT signaling pathway (Peltier et al. 2007, Srivastava et al. 2018, Zhang et al. 2018). In summary, this comprehensive transcriptome analysis revealed similarity between the H19-knockdown and VGF-knockdown groups and proposed that the PI3K-AKT signaling pathway and CBP may be pivotal factors in H19-induced carcinogenesis.

Table 2 Correlation of VGF expression in pNEN with clinicopathologic features.

\begin{tabular}{|c|c|}
\hline Clinicopathologic features & VGF low expression $(n=18)$ \\
\hline Age & $49.4 \pm 11.1$ \\
\hline Female & 13 \\
\hline Symptoms & 9 \\
\hline \multicolumn{2}{|l|}{ Location } \\
\hline Head & 7 \\
\hline Body and tail & 11 \\
\hline Functional & 3 \\
\hline Size $(\mathrm{cm})$ & $3.8 \pm 2.0$ \\
\hline Lymph node metastasis & 0 \\
\hline Local invasion & 0 \\
\hline Microvascular invasion & 0 \\
\hline Synchronous liver metastasis & 0 \\
\hline Ki67 (\%) & $3.8 \pm 3.2$ \\
\hline \multicolumn{2}{|l|}{ Grading } \\
\hline G1 & 9 \\
\hline $\mathrm{G} 2$ & 9 \\
\hline G3 & 0 \\
\hline \multicolumn{2}{|l|}{$\mathrm{T}$} \\
\hline $\mathrm{T} 1$ & 2 \\
\hline $\mathrm{T} 2$ & 10 \\
\hline T3 & 6 \\
\hline T4 & 0 \\
\hline \multicolumn{2}{|l|}{$\mathrm{N}$} \\
\hline NO & 18 \\
\hline N1 & 0 \\
\hline \multicolumn{2}{|l|}{$\mathrm{M}$} \\
\hline MO & 18 \\
\hline M1 & 0 \\
\hline \multicolumn{2}{|l|}{ TNM stage } \\
\hline I & 2 \\
\hline II & 16 \\
\hline III & 0 \\
\hline IV & 0 \\
\hline Tumor progression & 0 \\
\hline Tumor-related death & 0 \\
\hline
\end{tabular}

\begin{tabular}{|c|c|}
\hline VGF high expression $(n=21)$ & $P$ value \\
\hline $55.0 \pm 10.2$ & 0.108 \\
\hline 11 & 0.204 \\
\hline \multirow[t]{2}{*}{13} & 0.455 \\
\hline & 0.718 \\
\hline \multicolumn{2}{|l|}{7} \\
\hline \multicolumn{2}{|l|}{14} \\
\hline 1 & 0.318 \\
\hline $5.2 \pm 2.9$ & 0.085 \\
\hline 7 & $0.010 *$ \\
\hline 7 & $0.010 *$ \\
\hline 4 & 0.110 \\
\hline 8 & $0.004 *$ \\
\hline \multirow{2}{*}{$8.5 \pm 7.8$} & $0.016 *$ \\
\hline & $0.011 *$ \\
\hline \multicolumn{2}{|l|}{2} \\
\hline \multicolumn{2}{|l|}{17} \\
\hline \multicolumn{2}{|l|}{2} \\
\hline \multicolumn{2}{|r|}{0.090} \\
\hline \multicolumn{2}{|l|}{1} \\
\hline \multicolumn{2}{|l|}{7} \\
\hline \multicolumn{2}{|l|}{8} \\
\hline \multicolumn{2}{|l|}{5} \\
\hline \multirow{2}{*}{\multicolumn{2}{|c|}{$0.010 *$}} \\
\hline & \\
\hline \multicolumn{2}{|l|}{7} \\
\hline & $0.001 *$ \\
\hline 13 & $0.004 *$ \\
\hline \multicolumn{2}{|l|}{8} \\
\hline \multirow{2}{*}{\multicolumn{2}{|c|}{$<0.001 *$}} \\
\hline & \\
\hline \multicolumn{2}{|l|}{6} \\
\hline \multicolumn{2}{|l|}{6} \\
\hline \multicolumn{2}{|l|}{8} \\
\hline 11 & $<0.001$ * \\
\hline 4 & 0.110 \\
\hline
\end{tabular}

VGF low expression (fold change $<10$ ); VGF high expression (fold change $\geq 10$ ). $\star P \leq 0.05, * * P \leq 0.01, * * * P \leq 0.001$.

https://erc.bioscientifica.com https://doi.org/10.1530/ERC-18-0552 c) 2019 Society for Endocrinology Published by Bioscientifica Ltd. Printed in Great Britain 

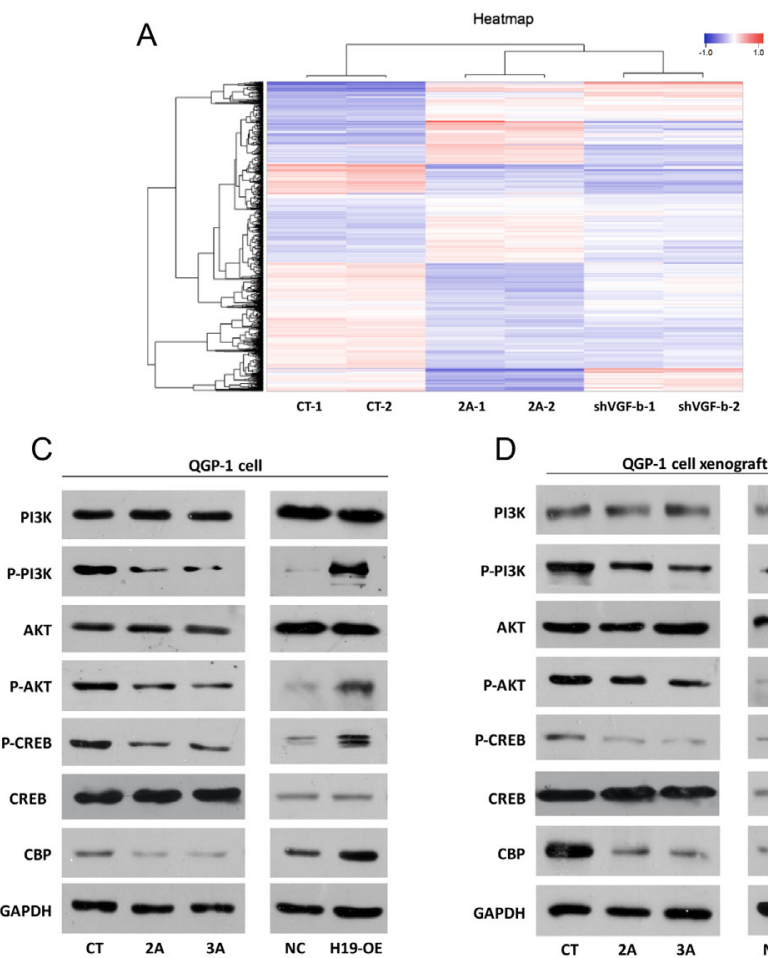

D

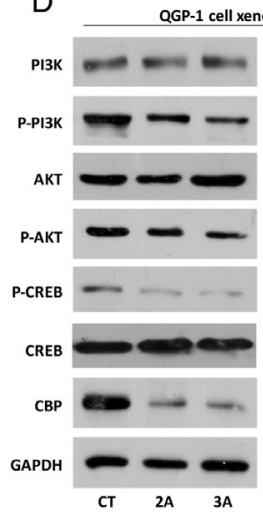

B

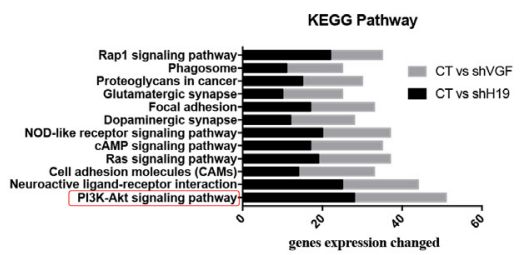

$E$
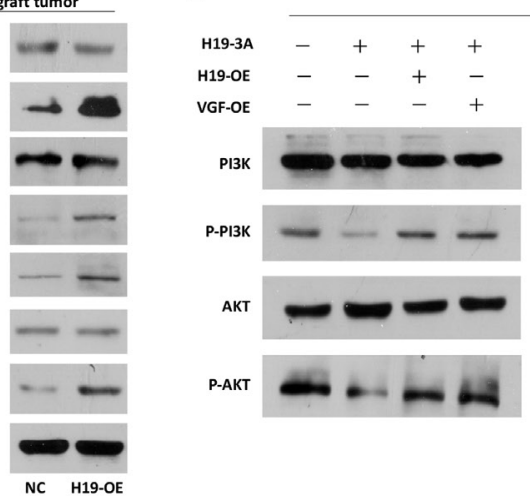

QGP-1 cell

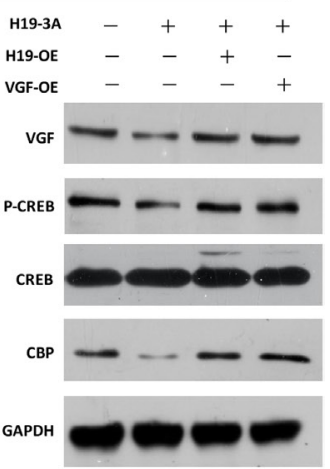

Figure 6

H19 interacts with and triggers VGF expression, which could activate the PI3K/AKT/CREB pathway in pNENs. (A) Heatmap of differentially expressed mRNAs in H19-knockdown cells, VGF-knockdown cells and control cells. (B) Enriched KEGG pathways in both differential gene sets. (C and D) Western blotting showing the protein expression of PI3K, P-PI3K, AKT, P-AKT, P-CREB, CREB and CBP in QGP-1 cells and xenografted tumors. (E) Western blotting showing that VGF-OE rescued the inhibitory effect of H19-3A on the expression of P-PI3K, P-AKT, P-CREB, and CBP. A full colour version of this figure is available at https://doi.org/10.1530/ERC-18-0552.

\section{H19 activated the PI3K/AKT/CREB pathway in pNENs via triggering and interacting with VGF}

Interestingly, we searched the literature and found that VGF could be induced by the PI3K/AKT/CREB signaling pathway (Rodriguez-Seoane et al. 2015). Researchers have already shown that the phosphorylation of CREB at Ser133 could induce VGF expression, which was also induced by activating the phosphorylation of AKT at Ser473 in mental disease (Ramos et al. 2014, RodriguezSeoane et al. 2015). Therefore, the effect of H19 on the activation of PI3K/AKT/CREB signaling in the progression of pNENs was subsequently determined.

The Western blotting results showed that the phosphorylation of AKT at Ser473, the phosphorylation of PI3K at Tyr458, the phosphorylation of CREB at Ser133 and CBP expression were decreased in QGP-1 cells when H19 was knocked down and were increased when H19 was overexpressed (Fig. 6C). Furthermore, Western blotting analysis identified similar results in the xenografted tumors with $\mathrm{H} 19$ silencing or overexpression (Fig. 6D). In addition, the combined transfection of H19-3A, H19-OE or VGF-OE was conducted to further assess protein expression in the PI3K/AKT/CREB pathway. We found that VGF-OE rescued the inhibitory effect of H19-3A on the expression of P-PI3K, P-AKT, P-CREB and CBP, which was consistent with the abovementioned results (Fig. 6E). Taken together, these data demonstrated that H19 activated the PI3K/AKT/CREB pathway in pNENs by triggering and interacting with VGF.

\section{Discussion}

lncRNAs can regulate gene expression in various ways, including chromatin remodeling and transcriptional and posttranscriptional processing (Fang \& Fullwood 2016). However, the understanding of the pathogenesis and prognostic implications of lncRNAs in neuroendocrine neoplasms is in its preliminary stages. One study thus far has described that menin activates lncRNA Meg3 via H3K4me3 and CpG hypomethylation at the Meg3 promoter CRE site to allow for the binding of the transcription factor CREB (Modali et al. 2015). Moreover, Meg3 exerts tumor suppressor activities in pNENs by (c) 2019 Society for Endocrinology Published by Bioscientifica Ltd. Printed in Great Britain 
blocking cell proliferation and delaying the cell cycle (Modali et al. 2015). This initial study of lncRNAs and pNENs suggested an important link between lncRNAs and NEN development, and a great deal of further study is necessary. Recent studies have highlighted the important roles of H19 during the complex process of tumorigenesis, starting from the early stages that involve translational deregulation and genomic instability through proliferation imbalance and stress management to metastasis (Raveh et al. 2015). Therefore, H19 is highly expressed in most tumors and is thought to be a carcinogenic gene with critical functions in tumorigenesis. To our knowledge, this study is the first report that generally investigates the expression, function, mechanism and clinical implication of H19 in pNENs.

When we detected the H19 expression level in pNEN patient samples, it was found that in patients with malignant behavior, H19 expression was upregulated, while H19 was downregulated in tumors with nonmalignant behavior. In addition, the H19 expression level was positively correlated with the degree of malignancy, especially in patients with liver metastasis. Our research mainly addresses the function and mechanism of H19 in pNENs with malignant behavior. Most pNENs are indolent but have malignant potential (Ro et al. 2013), but the study of pNENs with nonmalignant behavior should not be neglected. Based on the importance of H19 in tumor occurrence, the downexpression of H19 in pNENs with nonmalignant behavior may exert certain functions, which deserves further study. However, due to the absence of nonmalignant pNEN cell lines in the world, the study of H19 in pNENs with nonmalignant behavior cannot be conducted for the time being.

VGF affects feeding, energy expenditure, locomotor activity, sympathetic nerve activity, gastrointestinal motility and secretion and pancreatic hormone secretion (Levi et al. 2004). Alternatively, VGF has a degree of similarity in its pattern of expression with proteins of the secretogranin-chromogranin family (Levi et al. 2004). Many papers have reported that chromogranin A is a serological prognostic marker for pNENs that can be used to predict outcomes in patients with resected pNENs (Shanahan et al. 2016). The value of serum chromogranin $\mathrm{A}$ is reported as a predictor of tumor burden, therapeutic response and nomogram-based survival in moderate nonfunctional pNENs with liver metastases (Chou et al. 2012, Nölting et al. 2012, Han et al. 2015, Tian et al. 2016). Based on the similarity between VGF and chromogranin A, we showed great interest in VGF after obtaining the RNA pulldown and MS results, which indicated that H19 might bind to VGF. The following study was carried out and confirmed our hypothesis. Previous studies on VGF mainly focused on diseases of the nervous system, but only a few reported tumor studies have been conducted involving ovarian cancer, urothelial cell carcinoma, breast cancer, lung cancer and neuroblastoma (Rossi et al. 1992, Brait et al. 2013, Annaratone et al. 2014, Hayashi et al. 2014, Hwang et al. 2017). However, there is only one study related to VGF in neuroendocrine tumors. The study focused on VGF expression in human endocrine cells during development, adulthood, hyperplasia and tumor development and found that VGF was present in endocrine cells early during development and adulthood and increased in the context of hyperplasia and tumors (Rindi et al. 2007). Therefore, to our knowledge, this study is the first to report the relationship between high expression of VGF in pNENs and tumor malignancy and to further elucidate the function and mechanism of VGF in promoting proliferation and metastasis.

Due to the low incidence of pancreatic neuroendocrine tumors, the limited number of samples available for study and the few cell lines accessible for research, basic scientific research on pNENs is progressing slowly. However, the incidence rate of the disease is increasing annually, the prognosis of patients with malignant tumors, especially liver metastasis, is poor, and the risk of death is also present. It is becoming increasingly important to explore the pathogenesis of the tumor and to find effective prognostic markers and drug targets.

\section{Conclusions}

Our study highlights the functions and mechanisms of lncRNA H19 in regulating pNEN progression and malignant behavior. Furthermore, VGF was shown to bind directly to H19, which was overexpressed in pNENs and was positively correlated with H19 expression. In addition, high H19 and VGF expression correlated with deeper primary invasion depth, positive lymph node metastasis and advanced TNM stage. Moreover, we demonstrate that H19 upregulation in QGP-1 cells activates the PI3K/AKT/ CREB pathway by triggering VGF. These findings suggest that H19 and VGF can be promising potential biomarkers and therapeutic targets in pNENs.

Ethics approval and consent to participate

The use of human specimens for research was approved by the Committee on Ethics of Biomedicine, Second Military Medical University. We obtained 
the patients' written informed consent and approval from the Committee on Ethics of Biomedicine, Second Military Medical University prior to the use of the specimens for research purposes.

All in vivo animal studies were approved by the Committee on Ethics of Shanghai Institute of Materia Medica and were conducted in compliance with the Institutional Animal Care and Use Committee (IACUC) guidelines of Shanghai Institute of Materia Medica. The IACUC approval numbers are 2017-01-DK-44 and 2018-02-DK-63. All animals received humane care in compliance with the Public Health Service Policy on Humane Care and Use of Laboratory Animals.

\section{Supplementary data}

This is linked to the online version of the paper at https://doi.org/10.1530/ ERC-18-0552.

\section{Declaration of interest}

The authors declare that there is no conflict of interest that could be perceived as prejudicing the impartiality of the research reported.

\section{Funding}

This work was supported by the Medical Guidance Project of Shanghai Science and Technology Commission (No. 124119a0501).

\section{Consent for publication}

Consent to publish has been obtained from all authors.

\section{Author contribution statement}

Conception and design of the study: Ji M, Shao C H. Generation, collection, assembly, analysis of data: Ji M, Yao Y L. Drafting and revision of the manuscript: Ji M, Yao Y L, Liu A A, Shi L G. Approval of the final version of the manuscript: all authors.

\section{Acknowledgements}

The authors thank Lixing Zhan and Meiyan Qi (Shanghai Institute of Nutrition and Health, Chinese Academy of Sciences) for the technical support of this study.

\section{References}

Annaratone L, Medico E, Rangel N, Castellano I, Marchio C, Sapino A \& Bussolati G 2014 Search for neuro-endocrine markers (chromogranin A, synaptophysin and VGF) in breast cancers. An integrated approach using immunohistochemistry and gene expression profiling. Endocrine Pathology 25 219-228. (https://doi.org/10.1007/ s12022-013-9277-4)

Bartonicek N, Maag JLV \& Dinger ME 2016 Long noncoding RNAs in cancer: mechanisms of action and technological advancements. Molecular Cancer 15 43. (https://doi.org/10.1186/s12943-016-0530-6)

Brait M, Maldonado L, Noordhuis MG, Begum S, Loyo M, Poeta ML, Barbosa A, Fazio VM, Angioli R, Rabitti C, et al. 2013 Association of promoter methylation of VGF and PGP9.5 with ovarian cancer progression. PLOS ONE 8 e70878. (https://doi.org/10.1371/journal. pone.0070878)

Chen XN \& Korenberg JR 1995 Localization of human crebbp (CREB binding-protein) to $16 \mathrm{p} 13.3$ by fluorescence in-situ hybridization. Cytogenetics and Cell Genetics 71 56-57. (https://doi. org/10.1159/000134062)

Chou WC, Hung YS, Hsu JT, Chen JS, Lu CH, Hwang TL, Rau KM, Yeh KY, Chen TC \& Sun CF 2012 Chromogranin A is a reliable biomarker for gastroenteropancreatic neuroendocrine tumors in an Asian population of patients. Neuroendocrinology 95 344-350. (https://doi.org/10.1159/000333853)

Fang YW \& Fullwood MJ 2016 Roles, functions, and mechanisms of long non-coding RNAs in cancer. Genomics, Proteomics and Bioinformatics 14 42-54. (https://doi.org/10.1016/j.gpb.2015.09.006)

Fitzgerald TL, Hickner ZJ, Schmitz M \& Kort EJ 2008 Changing incidence of pancreatic neoplasms - a 16-year review of statewide tumor registry. Pancreas 37 134-138. (https://doi.org/10.1097/ MPA.0b013e318163a329)

Gao LM, Natov NS, Daly KP, Masud F, Chaudhry S, Sterling MJ \& Saif MW 2018 An update on the management of pancreatic neuroendocrine tumors. Anti-Cancer Drugs 29 597-612. (https://doi. org/10.1097/CAD.0000000000000633)

Han X, Zhang CY, Tang M, Xu XF, Liu LX, Ji Y, Pan BS \& Lou WH 2015 The value of serum chromogranin A as a predictor of tumor burden, therapeutic response, and nomogram-based survival in wellmoderate nonfunctional pancreatic neuroendocrine tumors with liver metastases. European Journal of Gastroenterology and Hepatology 27 527-535. (https://doi.org/10.1097/MEG.0000000000000332)

Hayashi M, Bernert H, Kagohara LT, Maldonado L, Brait M, Schoenberg M, Bivalacqua T, Netto GJ, Koch W, Sidransky D, et al. 2014 Epigenetic inactivation of VGF associated with urothelial cell carcinoma and its potential as a non-invasive biomarker using urine. Oncotarget 5 3350-3361. (https://doi.org/10.18632/ oncotarget.1949)

Hickey CJ, Kim JH \& Ahn EYE 2014 New discoveries of old son: a link between RNA splicing and cancer. Journal of Cellular Biochemistry $\mathbf{1 1 5}$ 224-231. (https://doi.org/10.1002/jcb.24672)

Hwang W, Chiu YF, Kuo MH, Lee KL, Lee AC, Yu CC, Chang JL, Huang WC, Hsiao SH, Lin SE, et al. 2017 Expression of neuroendocrine factor VGF in lung cancer cells confers resistance to EGFR kinase inhibitors and triggers epithelial-to-mesenchymal transition. Cancer Research 77 3013-3026. (https://doi. org/10.1158/0008-5472.CAN-16-3168)

Ito T, Igarashi H \& Jensen RT 2012 Therapy of metastatic pancreatic neuroendocrine tumors (pNETs): recent insights and advances. Journal of Gastroenterology 47 941-960. (https://doi.org/10.1007/ s00535-012-0642-8)

Jiang C, Lin WJ, Sadahiro M, Labonte B, Menard C, Pfau ML, Tamminga CA, Turecki G, Nestler EJ, Russo SJ, et al. 2018 VGF function in depression and antidepressant efficacy. Molecular Psychiatry 23 1632-1642. (https://doi.org/10.1038/mp.2017.233)

Kaku M, Nishiyama T, Yagawa K \& Abe M 1980 Establishment of a carcinoembryonic antigen-producing cell-line from human pancreatic-carcinoma. Gan 71 596-601.

Keniry A, Oxley D, Monnier P, Kyba M, Dandolo L, Smits G \& Reik W 2012 The H19 lincRNA is a developmental reservoir of miR-675 that suppresses growth and Igf1r. Nature Cell Biology 14 659-665. (https:// doi.org/10.1038/ncb2521)

Lawrence B, Gustafsson BI, Chan A, Svejda B, Kidd M \& Modlin IM 2011 The epidemiology of gastroenteropancreatic neuroendocrine tumors. Endocrinology and Metabolism Clinics of North America $\mathbf{4 0}$ 1-18, vii. (https://doi.org/10.1016/j.ecl.2010.12.005)

Levi A, Ferri GL, Watson E, Possenti R \& Salton SRJ 2004 Processing, distribution, and function of VGF, a neuronal and endocrine peptide precursor. Cellular and Molecular Neurobiology 24 517-533. (https:// doi.org/10.1023/B:CEMN.0000023627.79947.22) 
Li CL, Li MM, Yu HJ, Shen XB, Wang JT, Sun X, Wang QW \& Wang C 2017 Neuropeptide VGF C-terminal peptide TLQP-62 alleviates lipopolysaccharide-lnduced memory deficits and anxiety-like and depression-like behaviors in mice: the role of BDNF/TrkB signaling. ACS Chemical Neuroscience 8 2005-2018. (https://doi.org/10.1021/ acschemneuro.7b00154)

Modali SD, Parekh VI, Kebebew E \& Agarwal SK 2015 Epigenetic regulation of the lncRNA MEG3 and its target c-MET in pancreatic neuroendocrine tumors. Molecular Endocrinology 29 224-237. (https://doi.org/10.1210/me.2014-1304)

Nölting S, Kuttner A, Lauseker M, Vogeser M, Haug A, Herrmann KA, Hoffmann JN, Spitzweg C, Göke B \& Auernhammer CJ 2012 Chromogranin A as serum marker for gastroenteropancreatic neuroendocrine tumors: a single center experience and literature review. Cancers 4 141-155. (https://doi.org/10.3390/cancers4010141)

Ohki R, Saito K, Chen Y, Kawase T, Hiraoka N, Saigawa R, Minegishi M, Aita Y, Yanai G, Shimizu H, et al. 2014 PHLDA3 is a novel tumor suppressor of pancreatic neuroendocrine tumors. PNAS $\mathbf{1 1 1}$ E2404-E2413. (https://doi.org/10.1073/pnas.1319962111)

Peltier J, O'Neill A \& Schaffer DV 2007 PI3K/AKT and CREB regulate adult neural hippocampal progenitor proliferation and differentiation. Developmental Neurobiology 67 1348-1361. (https:// doi.org/10.1002/dneu.20506)

Proye C 2001 Natural history of liver metastasis of gastroenteropancreatic neuroendocrine tumors: place for chemoembolization. World Journal of Surgery 25 685-688. (https:// doi.org/10.1007/s00268-001-0013-8)

Ramos A, Rodriguez-Seoane C, Rosa I, Trossbach SV, Ortega-Alonso A, Tomppo L, Ekelund J, Veijola J, Jarvelin MR, Alonso J, et al. 2014 Neuropeptide precursor VGF is genetically associated with social anhedonia and underrepresented in the brain of major mental illness: its downregulation by DISC1. Human Molecular Genetics $\mathbf{2 3}$ 5859-5865. (https://doi.org/10.1093/hmg/ddu303)

Raveh E, Matouk IJ, Gilon M \& Hochberg A 2015 The H19 long noncoding RNA in cancer initiation, progression and metastasis - a proposed unifying theory. Molecular Cancer 14 184. (https://doi. org/10.1186/s12943-015-0458-2)

Rindi G, Licini L, Necchi V, Bottarelli L, Campanini N, Azzoni C, Favret M, Giordano G, D'Amato F, Brancia C, et al. 2007 Peptide products of the neurotrophin-inducible gene VGF are produced in human neuroendocrine cells from early development and increase in hyperplasia and neoplasia. Journal of Clinical Endocrinology and Metabolism 92 2811-2815. (https://doi.org/10.1210/jc.2007-0035)

Ro C, Chai WX, Yu VE \& Yu R 2013 Pancreatic neuroendocrine tumors: biology, diagnosis, and treatment. Chinese Journal of Cancer 32 312-324. (https://doi.org/10.5732/cjc.012.10295)

Rodriguez-Seoane C, Ramos A, Korth C \& Requena JR 2015 DISC1 regulates expression of the neurotrophin VGF through the PI3K/ AKT/CREB pathway. Journal of Neurochemistry 135 598-605. (https:// doi.org/10.1111/jnc.13258)

Rossi A, Granata F, Augustitocco G, Canu N, Levi A \& Possenti R 1992 Expression in murine and human neuroblastoma cell-lines of VGF, a tissue specific protein. International Journal of Developmental Neuroscience 10 527-534. (https://doi.org/10.1016/07365748(92)90053-3)

Sadahiro M, Erickson C, Lin WJ, Shin AC, Razzoli M, Jiang C, Fargali S, Gurney A, Kelley KA, Buettner C, et al. 2015 Role of VGF-derived carboxy-terminal peptides in energy balance and reproduction: analysis of 'humanized' knockin mice expressing full-length or truncated VGF. Endocrinology 156 1724-1738. (https://doi. org/10.1210/en.2014-1826)

Salton SRJ, Fischberg DJ \& Dong KW 1991 Structure of the gene encoding VGF, a nervous system-specific mRNA that is rapidly and selectively induced by nerve growth-factor in PC12 cells. Molecular and Cellular Biology 11 2335-2349. (https://doi.org/10.1128/ MCB.11.5.2335)

Shanahan MA, Salem A, Fisher A, Cho CS, Leverson G, Winslow ER \& Weber SM 2016 Chromogranin A predicts survival for resected pancreatic neuroendocrine tumors. Journal of Surgical Research 201 38-43. (https://doi.org/10.1016/j.jss.2015.10.006)

Sibilia V, Pagani F, Bulgarelli I, Mrak E, Broccardo M, Improta G, Severini C, Possenti R \& Guidobono F 2010 TLQP-21, a VGF-derived peptide, prevents ethanol-induced gastric lesions: insights into its mode of action. Neuroendocrinology 92 189-197. (https://doi. org/10.1159/000319791)

Srivastava P, Dhuriya YK, Kumar V, Srivastava A, Gupta R, Shukla RK, Yadav RS, Dwivedi HN, Pant AB \& Khanna VK 2018 PI3K/AKT/GSK3 beta induced CREB activation ameliorates arsenic mediated alterations in nmda receptors and associated signaling in rat hippocampus: neuroprotective role of curcumin. Neurotoxicology 67 190-205. (https://doi.org/10.1016/j.neuro.2018.04.018)

Thakker-Varia S \& Alder J 2009 Neuropeptides in depression: role of VGF. Behavioural Brain Research 197 262-278. (https://doi. org/10.1016/j.bbr.2008.10.006)

Tian TT, Gao J, Li N, Li YY, Lu M, Li ZW, Lu ZH, Li J \& Shen L 2016 Circulating chromogranin $\mathrm{A}$ as a marker for monitoring clinical response in advanced gastroenteropancreatic neuroendocrine tumors. PLOS ONE 11 e0154679. (https://doi.org/10.1371/journal. pone.0154679)

Wang SH, Ma F, Tang ZH, Wu XC, Cai Q, Zhang MD, Weng MZ, Zhou D, Wang JD \& Quan ZW 2016 Long non-coding RNA H19 regulates FOXM1 expression by competitively binding endogenous miR-342-3p in gallbladder cancer. Journal of Experimental and Clinical Cancer Research 35 160. (https://doi.org/10.1186/s13046016-0436-6)

Yang F, Bi JW, Xue XC, Zheng LM, Zhi KK, Hua JD \& Fang GE 2012 Up-regulated long non-coding RNA H19 contributes to proliferation of gastric cancer cells. FEBS Journal 279 3159-3165. (https://doi. org/10.1111/j.1742-4658.2012.08694.x)

Yang GD, Lu XZ \& Yuan LJ 2014 lncRNA: a link between RNA and cancer. Biochimica et Biophysica Acta 1839 1097-1109. (https://doi. org/10.1016/j.bbagrm.2014.08.012)

Yohanathan L, Dossa F, St Germain AT, Golbafian F, Moulton CA, McGilvray ID, Greig PD, Serra S, Wei AC, Jhaveri KS, et al. 2019 Management and surveillance of non-functional pancreatic neuroendocrine tumours: retrospective review. Pancreatology 19 360-366. (https://doi.org/10.1016/j.pan.2019.02.008)

Yoshimizu T, Miroglio A, Ripoche MA, Gabory A, Vernucci M, Riccio A, Colnot S, Godard C, Terris B, Jammes H, et al. 2008 The H19 locus acts in vivo as a tumor suppressor. PNAS 105 12417-12422. (https:// doi.org/10.1073/pnas.0801540105)

Zhang W, Song JK, Yan R, Li L, Xiao ZY, Zhou WX, Wang ZZ, Xiao W \& Du GH 2018 Diterpene ginkgolides protect against cerebral ischemia/ reperfusion damage in rats by activating Nrf2 and CREB through PI3K/Akt signaling. Acta Pharmacologica Sinica 39 1259-1272. (https://doi.org/10.1038/aps.2017.149)

Received in final form 9 April 2019

Accepted 30 April 2019 https://erc.bioscientifica.com https://doi.org/10.1530/ERC-18-0552 (c) 2019 Society for Endocrinology Published by Bioscientifica Ltd. Printed in Great Britain 